\title{
Conducting Polymer Actuators for Medical Devices and Cell Mechanotransduction
}

\author{
Edwin Jager
}

Linköping University Post Print

\section{\$ Tweet}

N.B.: When citing this work, cite the original article.

(C2013 IEEE. Personal use of this material is permitted. However, permission to reprint/republish this material for advertising or promotional purposes or for creating new collective works for resale or redistribution to servers or lists, or to reuse any copyrighted component of this work in other works must be obtained from the IEEE.

Edwin Jager, Conducting Polymer Actuators for Medical Devices and Cell Mechanotransduction, 2013, IEEE/ASME International Conference on Advanced Intelligent Mechatronics, 2013, 16611666.

Postprint available at: Linköping University Electronic Press

http://urn.kb.se/resolve?urn=urn:nbn:se:liu:diva-99434 


\title{
Conducting Polymer Actuators for Medical Devices and Cell Mechanotransduction*
}

\author{
Edwin W.H. Jager
}

\begin{abstract}
Actuators made of conjugated polymers such as polypyrrole are interesting candidates as active elements in medical devices since they can be fabricated in small sizes and operated in saline solutions. In addition they can be microfabricated and integrated on silicon chips for instance for lab-on-a-chip and cell biology applications. Here, devices comprising polypyrrole (PPy) microactuators for mechanical stimulation of single cells are presented. In addition, novel interfacing and patterning methods for conjugated polymer (micro-) actuators are reported that open up for enhanced functionality and increased complexity of micromanipulators and microrobotics for instance for biomedicine.
\end{abstract}

\section{INTRODUCTION}

There is a continuous need to improve medical devices in order to advance current treatments or enable new therapies. One specific route taken is the use of minimal invasive therapies and robot-assisted surgery. These require smaller and more intelligent tools. Likewise in fundamental cell biology studies novel assays employing new technology allow generation of new knowledge and insights. In both cases advances in mechatronics, electroactive materials and actuator technology progress the development. One such technology is electroactive polymers [1].

\section{ACtUATION In CONDUCTING POLYMERS}

\section{A. Volume Change}

One class of EAPs is conjugated or conducting polymers (CP). These materials can be electrochemically oxidized and reduced which may result in a volume change of the material due to the insertion and extraction of counter ions into the CP. Depending on the dopants embedded in the polymer, two different redox reactions (and accompanying ion flows) are possible that can be generalized as (1) and (2) [1-3]: For a conjugated polymer $\mathrm{P}$ doped with small, mobile anions $\left(\mathrm{a}^{-}\right)$in contact with an electrolyte containing both mobile cations and anions the reaction is:

$$
\mathrm{P}^{+}\left(\mathrm{a}^{-}\right)+\mathrm{e}^{-} \leftrightarrow \mathrm{P}^{0}+\mathrm{a}^{-}(\mathrm{aq})
$$

*Research supported by European Science Foundation COST Action MP1003 ESNAM (European Scientific Network for Artificial Muscles) and COST-STSM-MP1003-8971 \& -13878, the Swedish Research Council (VR - 2010-6672), the Knut \& Alice Wallenberg Stiftelse (LiU-2010-00318, LiU-2012-01361), Swedish Foundation for Strategic Research (SSF), Linköping University, the EU Erasmus program, and the Australian Research Council for partial financial assistance through the Centers of Excellence, Discovery Projects (DP0878931) and Fellowships.

E. W. H. Jager is with Linköping University, Department of Physics, Chemistry and Biology, Biosensors and Bioelectronics Centre, 58183 Linköping, Sweden (phone:+46-13-281246; e-mail: edwin.jager@ liu.se).
That is, when reducing the polymer to its neutral state, anions $\mathrm{a}^{-}$are expelled and when oxidizing the polymer anions are inserted into the polymer matrix in order to compensate for the charge imbalance. On the other hand, for a polymer $\mathrm{P}$ doped with large, immobile anions $\mathrm{A}^{-}$in contact with an electrolyte containing small mobile cations $\mathrm{M}^{+}$the reaction is:

$$
\mathrm{P}^{+}\left(\mathrm{A}^{-}\right)+\mathrm{M}^{+}(\mathrm{aq})+\mathrm{e}^{-} \leftrightarrow \mathrm{P}^{0}\left(\mathrm{~A}^{-} \mathrm{M}^{+}\right)
$$

That is, cations $\mathrm{M}^{+}$are inserted when the polymer is reduced and expelled when the polymer is oxidized. In the former case the CP typically expands in the oxidized state, i.e. when a positive potential is applied, and in the latter case the $\mathrm{CP}$ expands in the reduced state, i.e. when a negative potential is applied.

CPs deliver high stresses, typically more than $5 \mathrm{MPa}$, exceeding that of mammalian skeletal muscles that deliver stresses of $0.35 \mathrm{MPa}$ [4]. The delivered strains of CPs are moderate, $1-10 \%$ compared to $\sim 20 \%$ strain of natural muscles. However, the volume change of PPy doped with dodecylbenzenesulphonate, $\mathrm{PPy}(\mathrm{DBS})$, is highly anisotropic, i.e. the perpendicular expansion is much higher (30-40\%) than the in-plane volume change (strain) $[5,6]$. Since the volume change is based on ion and solvent exchange, delivered speeds are low, up to a few hundreds Hz.

\section{B. PPy Actuators}

This volume change can be employed to construct actuators in a variety of ways as illustrated in Fig. 1: One can
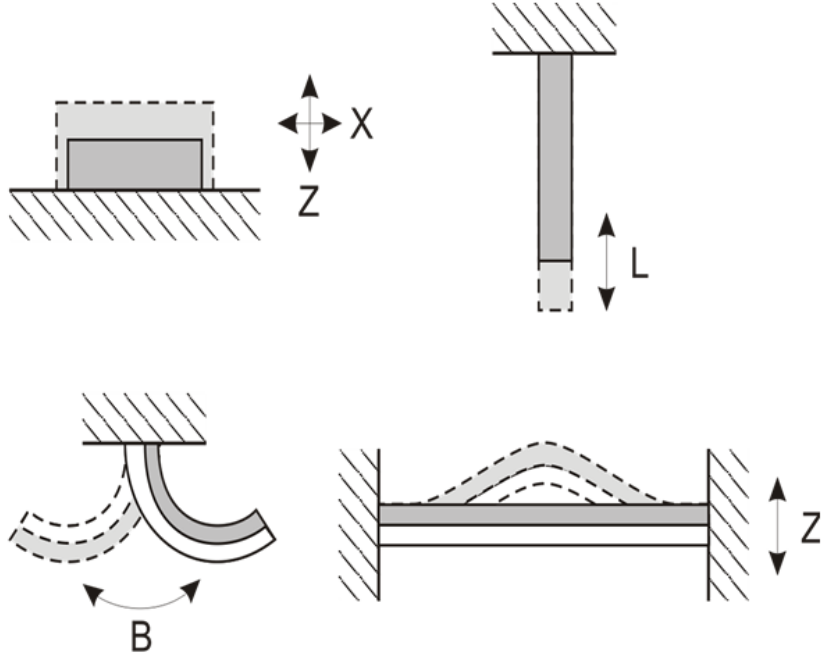

Figure 1 Different actuation modes used for PPy (micro-) actuators: perpendicular expansion, linear strain, bending bilayer, and buckling sheet. 
use the bulk volume change to drive an object in a piston-like motion. One can make sheets, tubes or fibers exploiting the strain. Alternatively, one can laminate the polymer to a second (or more) layer and convert the volume change into a bending or buckling motion depending on how the bilayer (or multilayer) is clamped. We have miniaturized the CP actuators to create microactuators $[7,8]$, including complex systems such as microrobots [9] by employing photolithography and microfabrication methods, modified to meet $\mathrm{CP}$ requirements.

\section{Mechanotransduction Devices}

\section{A. Mechanotransduction in Cells}

The effects of biochemical cues on cellular development are widely known. However, the importance of physical and mechanical influences on cell behaviour has only recently been acknowledged. Mechanotransduction is not only important for functioning of sensory cells, e.g. inner hair cells of the cochlea, but affects a wide range of cell types including myocytes, endothelial cells, and vascular smooth muscle cells. Mechanical stimuli have been shown to affect cellular physiology and behaviour, e.g. via intra cellular changes such as calcium concentrations and signalling pathways [10]. These stimuli are important for cell division, differentiation, motility, embryonic development and sensing $[11,12]$, and the lack thereof might be a factor in various diseases [10]. Cells receive large-scale mechanical signals, e.g. from gravitational forces, muscle contractions or fluid pressure (blood vessels, bladder) that are transduced from the tissue, via the extra cellular matrix through integrin receptors in the cell membrane that are coupled to the actin cytoskeleton, triggering cellular pathways. In addition, cells may receive mechanical stimuli from neighbouring cells or fluid flow, e.g. via cadherins and stretch activated ion channels. However, many details on how mechanical stress triggers intracellular signalling pathways are still unknown $[13,14]$.

Mechanotransduction is well studied in organs and tissues, for instance by using flexible tissue culture substrates that can be stretched by external means such as the FlexCell $^{\mathrm{TM}}$ system. On the cellular and multicellular level, there is a lack of devices that can apply physically relevant
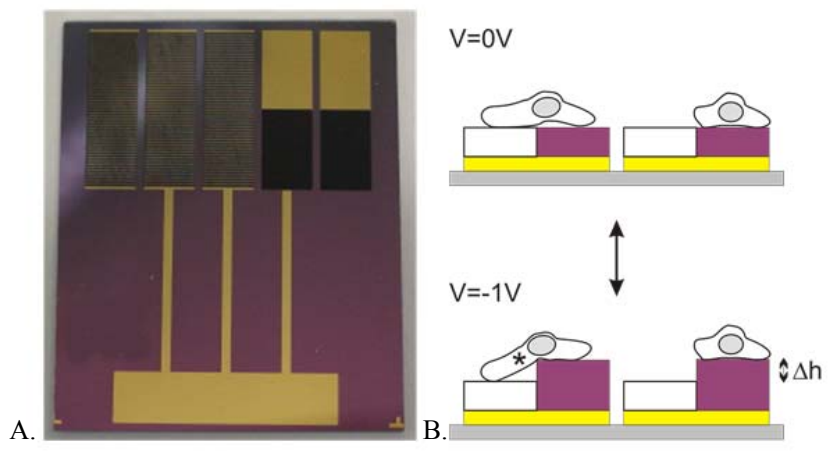

Figure 2 A. Mechanostimulation chip $2 \times 2.5 \mathrm{~cm}^{2}$ comprising two actuator areas (A) and three control areas (C1-C3). B. Upon application of a potential, the PPy actuator A will expand vertically and thus stretch cells that are situated along the borders, i.e. that adhere to both the PPy and the passive polymer. Cells that are located on only PPy or the passive polymer (C2) are not mechanically stimulated. C. Intracellular Ca ${ }^{2+}$ recordings of several cells on the microactuator A (solid lines) and recordings from several cells on the active control area C2 (dashed line).

\section{B. Mechanostimulation chips}

Since PPy can operate in aqueous salt solutions including cell culture media, it can be used to drive microactuators that can mechanically stimulate (single) cells. We are currently developing such devices for cellular mechanostimulation [15].

A mechanostimulation device that we developed is a microfabricated chip that comprised PPy microactuators, designed as alternating lines of PPy(DBS) and the passive structural material SU-8, both $100 \mu \mathrm{m}$ wide, and $11.0 \pm 0.6$ $\mu \mathrm{m}$ and $10.0 \mu \mathrm{m}$ thick respectively ("A" in Fig. 2A) [16]. The actuators are activated by a small a potential of $-1 \mathrm{~V}$ vs $\mathrm{Ag} / \mathrm{AgCl}$ for 5 mins causing the PPy lines to expand perpendicularly $(1.0 \pm 0.4 \mu \mathrm{m}$ or $8.8 \pm 3.7 \%$, in cell culture media) and thus mechanically stimulate cells that are situated along the borders, i.e. that adhere to both the PPy and SU-8 (Fig. 2B). On the other hand, cells that are positioned on only PPy or SU-8 are not mechanically stimulated. Also three different control areas $\mathrm{C} 1$ to $\mathrm{C} 3$ were integrated on the chip. The control area $\mathrm{Cl}$ is a passive patterned area identical to the microactuator A, but not electrically addressed, and acts as a control for the effect of the material and topography on the seeded cells. The control area $\mathrm{C} 2$ is a planar area of both PPy and SU8. Cells adhered on the PPy surface will receive the same electrochemical stimulation as cells on the microactuator A, but will not be mechanically stimulated. $\mathrm{C} 3$ is a passive area of planar PPy and SU8 identical to C2 but not electrically addressed and is used to investigate passive surface effects on the cells.

We have seeded renal epithelial (MDCK) cells which are responsive to mechanical stimuli and physiologically relevant, on top of the mechanostimulation chip and let them adhere overnight. Thereafter they were loaded with the $\mathrm{Ca}^{2+}$ dye Fura-2 so that we could follow the cellular response to the mechanostimulation in real time. The MDCK cells showed good adhesion and spreading on the PPy and SU8 surfaces. Next, we mechanically stimulated individual cells by activating the PPy-microactuators for $300 \mathrm{~s}$ at $-1.0 \mathrm{~V}$ followed by $300 \mathrm{~s}$ at $0.0 \mathrm{~V}$. This mechanical stimulation resulted in an increase in intracellular $\mathrm{Ca}^{2+}$ of the cells adhered to the actuators (Fig. 2C). The intracellular $\mathrm{Ca}^{2+}$

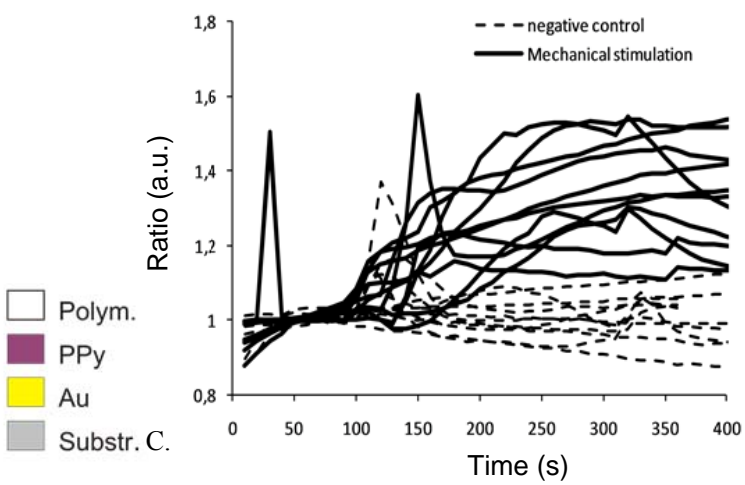


increase was slow but followed to the expansion profile of PPy-microactuators well. The gradual intracellular $\mathrm{Ca}^{2+}$ increase was absent in the cells that adhered on the active PPy area $\mathrm{C} 2$, thus further indicating that the $\mathrm{Ca}^{2+}$ signal was indeed mechanically induced. We were further able to establish that this $\mathrm{Ca}^{2+}$ response was caused by an autocrine ATP signalling pathway associated with mechanical stimulation of the cells, one of the most important mechanosensing mechanisms in MDCK cells.

\section{MEDiCAL DEVICES}

As mentioned CPs provide interesting possibilities to drive medical devices. A variety of examples have been presented.

\section{A. Microanastomosis Connector}

The microanastomosis connector was medical device developed by Micromuscle AB to reconnect both endings of a small, 1-3 mm diameter blood vessel, separated either by trauma or by surgery, as an alternative to micro-suturing [17]. The device was formed as a rolled-up tube, made of a three layers: an inner blood-compatible polyurethane layer; a micropatterned $\mathrm{Au}$ layer to predetermine the bending direction [18] and carry the current; and PPy(DBS) as the active layer. Applying a small potential caused the tube to shrink by rolling up the sheet more tightly. Once inserted in both ends of a cut blood vessel, the potential was reversed and the tube expanded by unrolling the sheet thus holding the two vessel ends together during the healing process. The materials and device passed basic biocompatibility testing, including cytotoxicity, irritation, acute systemic toxicity, and hemolysis. A small implantation study of the connector in a rat model was also performed. After three months, the devices showed not to be causing blood clotting or other obstruction, indicating blood- and biocompatibility. Coagulation studies using a Chandler looping setup were also performed on both heparinized (in order to increase blood compatibility) and untreated, as fabricated PPy connectors. These studies showed that there was no difference between the heparinized and unheparinized PPy connectors and that they were as good as commercial stents, with regards to coagulation.

\section{B. Steerable Guide Wires and Catheters}

Small size and easy addressing make CPs an interesting material to use as the active material to deflect guide wires and catheters thus aiding the navigation through the vascular system. One design strategy has been to assemble two or four strips/actuators on opposing sides of the wire/catheter in
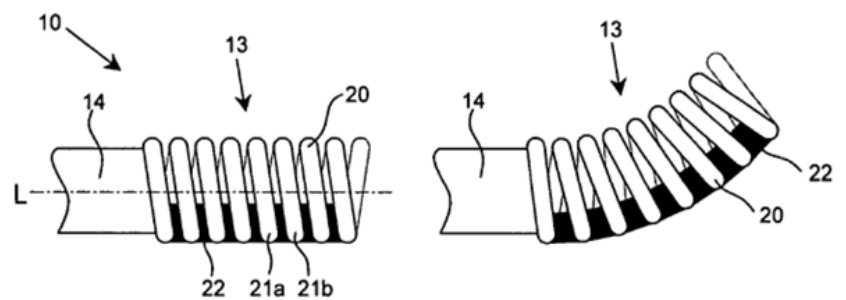

Figure 3 A schematic drawing of the principle of the PPy-activated guide wire tip for aided navigation in blood vessels. Left, PPy is in the contracted state and the guide wire is straight. Right, PPy is in the expanded state and the guide wire tip bends. Reproduced from Krogh and Jager, WO2007057132 order to achieve bending in both $\mathrm{x}$ and $\mathrm{y}$ direction or circular motion of the tip, as for instance demonstrated by Lee et al. [19]. They altered a standard $0.5 \mathrm{~mm}$ outer diameter catheter by applying one or two opposing pairs of PPy electrodes on the tip and were able to achieve bending curvatures up to $0.06 \mathrm{~m}^{-1}$ when activating in a (non-physiological) $1 \mathrm{M} \mathrm{NaPF}_{6}$ solution.

Krogh and Jager demonstrated an easier design strategy that also functioned in a saline solution [20]. They applied PPy(DBS) only on one side of the coil tip of a guide wire (Fig. 3). The volume changes resulted in a bending of the tip, due to the asymmetric application of PPy, similar to the bending beam principle. A circular motion of the tip can be achieved by rotating the guide wire as is common clinical practice using standard fixed curvature guide wires. This design makes electronic control and device fabrication extremely easy since only one PPy actuator has to be integrated on the tip. The counter electrode can be placed nearby or integrated as an antagonistic actuator.

Likewise, there is a need to actively alter the curvature of cochlear implants during the surgical insertion. For this application, Zhou and co-workers developed a cochlear electrode array that can electroactively bend using PPy [21]. The active element consisted of a PPy/Pt/PVDF/Pt/PPy multilayer actuator, with the PVDF pores filled with a propylene carbonate-based electrolyte. The multilayer actuator was mounted on the back of a standard cochlear electrode array and bending of almost $180^{\circ}$ could be achieved.

\section{Balloon Catheter}

Gumm developed a specialized rotating balloon system for bifurcation stenting (i.e. positioning two stents around a bifurcation, one stent in the main artery and one in the side branch using a two-guide wire system) [22]. The system required the pivot points at both ends of the balloon to be sealed on demand in order to mechanically lock the balloon in a fixed position, preventing rotation, and to be able to build up a liquid pressure to unfold the balloon and deploy the stent. Micromuscle AB and Boston Scientific developed a valve/seal designed as a thick PPy ring mounted on the balloon shaft under the pivot point of the dilation balloon [23]. The PPy ring could expand up to $40 \%$ of the PPy layer, sufficient to close the gap between the shaft and balloon pivot point, locking the balloon in place and preventing rotation. Also, the PPy ring was able to seal the balloon from leaking and could withstand inflation pressures up to $24 \mathrm{~atm}$, exceeding the clinically used pressures of 12-20 atm.

Standard ethylene oxide sterilization, as used in the medical device industry, did not affect the performance of the PPy valve/seal. Optimisation of the synthesis and activation conditions resulted in a maximum expansion of 15 $\mu \mathrm{m}$ for a $50 \mu \mathrm{m}$ thick PPy ring and an increased expansion speed (11 $\mu \mathrm{m}$ expansion the first few seconds) [24].

\section{INDIVIDUALly AdDRESSABLE PPy MicROACTUATORS}

In order to advance the use of $\mathrm{CP}$ actuators in medical devices e.g. in micromanipulation and microsurgery we need to increase the complexity of the devices. This requires the development of CP microactuators that can be individually 
Top view

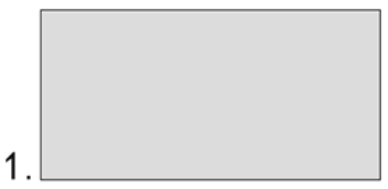

2.

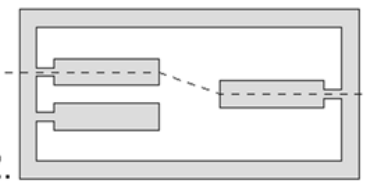

3

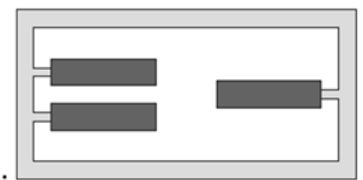

4

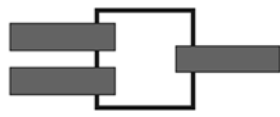

Figure 4 Patterning of the PPy tri-layer actuator. 1. Au (light grey) sputtering on both sides of the PVDF (white), sequentially. 2. Wet chemical etching of the Au electrodes including conducting frame. 3. Electrochemical deposition of PPy (dark grey) on both sides simultaneously. 4. The actuator unit is cut out from the PVDF membrane and conducting frame.

addressed and operated independently from the surrounding electrolyte. Patterning and interfacing are major obstacles in their development. Recently, we have developed an easy interface that can drive PPy tri-layer actuators and we devised microfabrication methods to pattern the actuators so that they could be individually addressed and operate in air [25]. The tri-layer microactuators, similar to the ones used for controlling the above mentioned Cochlear implant [21], comprise two outer PPy layers as the active layers and an inner porous separator of poly(vinylidene fluoride) (PVDF) containing the liquid electrolyte (lithium triflouromethanesulfonimide $\left(\mathrm{Li}^{+} \mathrm{TFSI}^{-}\right)$in propylene carbonate, $\left.\mathrm{PC}\right)$ $[26,27]$. When a potential difference is applied between the two PPy layers, one layer is oxidized while the other is reduced. The oxidized layer expands while the reduced layer contracts according to (1), resulting in a bending motion.

\section{A. Patterning Tri-layer Actuators}

We fabricated the patterned tri-layer milliactuators using microfabrication technologies (Fig. 4) [25]. First, we sputtered 20-30 nm Au on both sides of a piece of the PVDF membrane. We patterned the Au coated PVDF using Ma P1275 thick film photoresist on both sides, sequentially. Next, we wet chemically etched the Au pattern, i.e. the actuator shapes, leads and contact pads, in a $\mathrm{KI} / \mathrm{I}_{2}$ solution, and thereafter stripped the photoresist. In addition we devised a lift-off process to pattern the Au: First, we patterned the photoresist on both sides of the plain PVDF membrane, sputtered the $\mathrm{Au}$ on both sides sequentially and thereafter patterned the $\mathrm{Au}$ using lift-off. We electrochemically deposited PPy on the patterned $\mathrm{Au}$ on both sides simultaneously at a constant current of $0.1 \mathrm{~mA} / \mathrm{cm}^{2}$ for $12 \mathrm{~h}$ either at $-18{ }^{\circ} \mathrm{C}$ or room temperature in a solution of $0.1 \mathrm{M}$

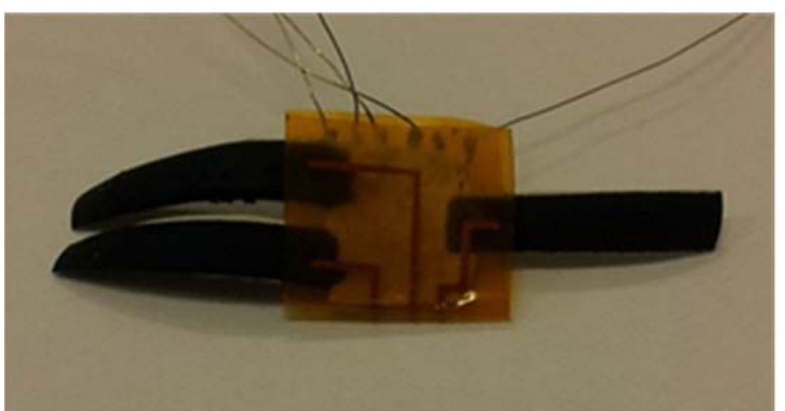

Figure $5 \mathrm{~A}$ photograph showing the $\mathrm{PVDF} / \mathrm{Au}$ actuator unit inside the folded FPCB interface and soldered wires. Each "finger" is $2 \times 10 \mathrm{~mm}^{2}$.

LiTFSi in PC with $1 \%$ DI water using an Ivium compactstat or IviumStat (Eindhoven, The Netherlands). Finally, we manually cut out the actuator unit in the desired final shape of a "hand" with 3 individually addressable "fingers", all monolithically integrated in a single piece of PVDF. The PPy fingers were $2 \mathrm{~mm}$ wide and $10 \mathrm{~mm}$ long of which $2 \mathrm{~mm}$ is positioned on the PVDF base in order to provide a contact pad for the electrical interface.

We compared the performance of a single microfabricated actuator with an unpatterned tri-layer actuator, i.e. manufactured as a large sheet and after PPy synthesis cut out by hand in the same shape as the microfabricated actuators. We could not notice any difference in device appearance between the two. The resulting driving currents and displacements of the two actuators were also similar. This shows that the microfabrication process has no negative effect on the device performance

\section{B. Interface}

Interfacing tri-layer microactuators has been an issue, especially with regards of short-circuiting the thin microactuators. Therefore, we developed a novel interface for CP actuators based on Flexible Printed Circuit Board (FPCB). With the FPCB interface we achieved a fine electrical contact that minimized short-circuiting. We were able to integrate an electrical circuit needed for individual control and future electrical components by etching the $\mathrm{Cu}$ contact pads and lines in a $\mathrm{HCl}$ and $\mathrm{H}_{2} \mathrm{O}_{2}(2: 1)$ bath. The FPCB is light-weight which makes future untethered control for instance in microrobotics a possibility. We designed the contacts to the top and the bottom electrodes of the tri-layer actuator to be made in a mirror image on the FPCB and put all the contact pads to the external electronics on one side. When we folded the FPCB interface, the top and bottom contacts automatically aligned and we could insert the actuator unit in between (Fig. 5). Unfortunately, the FPCM fold was slightly too flexible and we needed to clamp the interface with two alligator clips (see Fig. 6) in order to achieve good mechanical and thus good electrical contact. We soldered six fine wires to the FPCB contact pads to connect the actuator unit to the potentiostat to allow for individual control.

We compared the performance of an actuator clamped and contacted using a Kelvin clip with the same actuator mounted in the FPCB interface in order to investigate the influence of the interface on the actuator performance of the 
actuator. Surprisingly the FPCB interface resulted in a higher current and larger displacement of the actuator tip.

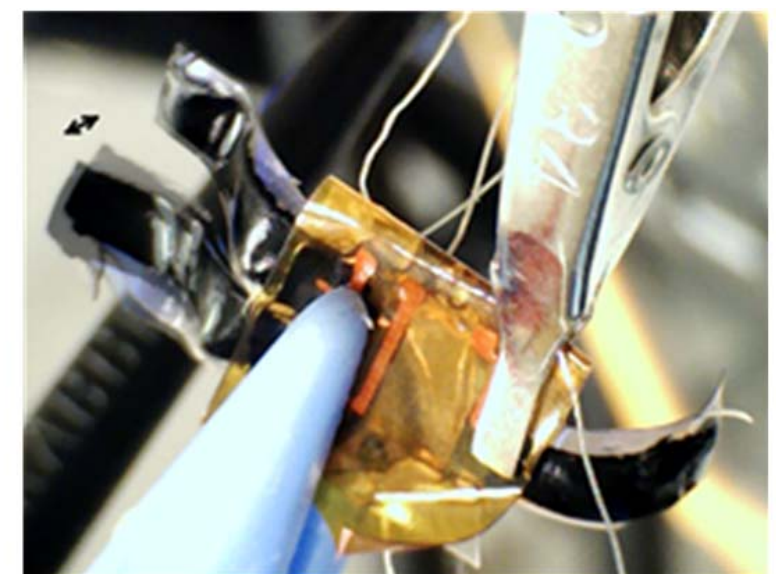

Figure 6 An overlay image of two frame grabs showing the actuation of the lower left finger (indicated by an arrow) of the assembled actuator device of Fig. 5. The other two fingers were not addressed and thus did not move. The PPy synthesis was not optimized so the deflection is only a few $\mathrm{mm}$.

\section{Individual control}

Fig. 6 shows an overlay image of two frame grabs of the mounted actuator device. We connected all six wires (one pair per actuator) to a UMUX unit of the Ivium potentiostat, connecting each top and bottom contact to a separate channel. The uMUX unit can only address each channel sequentially, this meant that we could address each finger individually, but only one at the time. We applied $\pm 2 \mathrm{~V}$ to all fingers, but used different pulse periods ( $1 \mathrm{~s}$ pulses for finger $1,2 \mathrm{~s}$ pulses for finger 2 and 5 s pulses for finger 3). As can be seen in Fig. 6 , we were able to address each finger individually, demonstrating successful microfabrication and interface connection to the individual actuating units.

Since we have solved the interfacing issues by developing an interface adapted for CPs as well as devised a photolithography based microfabrication process, we are currently working on downscaling the actuator to the microdomain. Thereafter we will develop more complex systems for medical devices and microrobotics comprising a number of individually controllable PPy microactuators.

\section{CONCLUSION}

Conjugated polymer materials and actuators have made huge progress since the first demonstrations in the early 90's and a variety of devices and prototypes have been demonstrated since. CPs and PPy in particular may have many applications in biomedicine, some of which have been presented here. We currently focus embedding the PPy microactuators in complex devices for mechanobiology, micromanipulators, and microrobotics.

\section{ACKNOWLEDGMENT}

E.W.H.J. wishes to thank his current and former coworkers at Micromuscle AB, University of Wollongong, and Linköping University without whom this work would not have been possible.

\section{REFERENCES}

[1] Q. Pei, O. Inganäs, "Electrochemical applications of the bending beam method. 1. Mass transport and volume changes in polypyrrole during redox," J. Phys. Chem., vol. 96, pp. 10507-10514, 1992.

[2] Q. Pei, O. Inganäs, "Electrochemical applications of the bending beam method. 2. Electroshrinking and slow relaxation in polypyrrole," J. Phys. Chem., vol. 97, pp. 6034-6041, 1993.

[3] M. R. Gandhi, P. Murray, G. M. Spinks, G. G. Wallace, "Mechanism of electromechanical actuation in polypyrrole," Synth. Met., vol. 73, pp. 247-256, 1995.

[4] J. D. Madden, R. A. Cush, T. S. Kanigan, I. W. Hunter, "Fast contracting polypyrrole actuators," Synth. Met., vol. 113, pp. 185$192,2000$.

[5] E. Smela, N. Gadegaard, "Surprising volume change in PPy(DBS): An atomic force microscopy study," Adv. Mater., vol. 11, pp. 953957, 1999.

[6] D. Melling, S. A. Wilson, M. Berggren, E. W. H. Jager, "Altering the structure of polypyrrole and the influence on electrodynamic performance," in Electroactive Polymer Actuators and Devices (EAPAD) San Diego, CA, USA, 2011, pp. 79760Z-1-13.

[7] E. Smela, O. Inganäs, I. Lundström, "Controlled folding of micrometer-size structures," Science, vol. 268, pp. 1735-1738, 1995

[8] E. W. H. Jager, E. Smela, O. Inganäs, "Microfabricating Conjugated Polymer Actuators," Science, vol. 290, pp. 1540-1545, 2000.

[9] E. W. H. Jager, O. Inganäs, I. Lundström, "Microrobots for Micrometer-Size Objects in Aqueous Media: Potential Tools for Single Cell Manipulation," Science, vol. 288, pp. 2335-2338, 2000.

[10] D. E. Jaalouk, J. Lammerding, "Mechanotransduction gone awry," Nat Rev Mol Cell Biol, vol. 10, pp. 63-73, 2009.

[11] M. A. Wozniak, C. S. Chen, "Mechanotransduction in development: a growing role for contractility," Nat Rev Mol Cell Biol, vol. 10, pp. 34-43, 2009.

[12] B. Geiger, J. P. Spatz, A. D. Bershadsky, "Environmental sensing through focal adhesions," Nat Rev Mol Cell Biol, vol. 10, pp. 21-33, 2009.

[13] N. Wang, J. P. Butler, D. E. Ingber, "Mechanotransduction across the cell surface and through the cytoskeleton," Science, vol. 260, pp. 1124-1127, 1993.

[14] F. J. Alenghat, J. D. Tytell, C. K. Thodeti, A. Derrien, D. E. Ingber, "Mechanical control of cAMP signaling through integrins is mediated by the heterotrimeric Galphas protein," J Cell Biochem, vol. 106, pp. 529-38, Mar 12009.

[15] E. W. H. Jager, K. Svennersten, V. Lundin, A. Richter-Dahlfors, M. Berggren, A. I. Teixeira, "Mechanotransduction in cells using polypyrrole microactuators," presented at the EuroEAP 2011, Pisa, Italy, 2011.

[16] K. Svennersten, M. Berggren, A. Richter-Dahlfors, E. W. H. Jager, "Mechanical stimulation of epithelial cells using polypyrrole microactuators," Lab on a Chip, vol. 11, pp. 3287 - 3293, 2011.

[17] C. Immerstrand, K. H. Peterson, K.-E. Magnusson, E. Jager, M. Krogh, M. Skoglund, A. Selbing, O. Inganäs, "Conjugated-Polymer Micro- and Milliactuators for Biological Applications," MRS bulletin, vol. 27, pp. 461-464, June 20022002.

[18] M. Krogh, O. Inganäs, E. Jager, "Fibre-reinforced microactuator," WO03039859, 2001.

[19] K. K. C. Lee, N. R. Munce, T. Shoa, L. G. Charron, G. A. Wright, J. D. Madden, V. X. D. Yang, "Fabrication and characterization of laser-micromachined polypyrrole-based artificial muscle actuated catheters," Sensors and Actuators A: Physical, vol. 153, pp. 230-236, 2009.

[20] M. Krogh, E. Jager, "Medical devices and methods for their fabrication and use," WO2007057132, 2005.

[21] D. Zhou, G. G. Wallace, G. M. Spinks, L. Liu, R. Cowan, E. Saunders, C. Newbold, "Actuators for the cochlear implant," Synth. Met., vol. 135, pp. 39-40, Apr 42003.

[22] D. Gumm, "Rotating stent delivery system for side branch access and protection and method of using same," WO03/017872, 2002.

[23] E. Jager, D. Carlsson, M. Krogh, M. Skoglund, "Electroactive polymer actuator devices and systems comprising such devices " WO2008113372 2007.

[24] D. Carlsson, E. Jager, M. Krogh, M. Skoglund, "Systems, device and object comprising electroactive polymer material, methods and uses relating to operation and provision thereof," WO2009038501, 2007. 
[25] E. W. H. Jager, N. Masurkar, N. F. Nworah, B. Gaihre, G. Alici, G. M. Spinks, "Patterning and electrical interfacing of individually controllable conducting polymer microactuators," submitted, 2013.

[26] G. Alici, V. Devaud, P. Renaud, G. Spinks, "Conducting polymer microactuators operating in air," J. Micromech. Microeng., vol. 19, p. $025017,2009$.

[27] Y. Wu, G. Alici, G. M. Spinks, G. G. Wallace, "Fast trilayer polypyrrole bending actuators for high speed applications," Synth. Met., vol. 156, pp. 1017-1022, 2006. 